\title{
Effect of EBG Structures for Reducing Noise in Multi-Layer PCBs for Digital Systems
}

Daehyun Chung, *Tae Hong Kim, Chunghyun Ryu, *Ege Engin, *Madhavan Swaminathan, and Joungho Kim

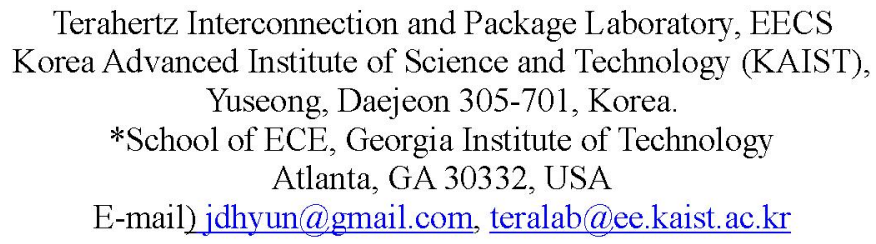

ABSTRACT- The effect of EBG structures in various noise source environments is investigated. In this paper a one-dimensional electromagnetic band gap structure (1D-EBG) has been used in the power/ground planes for isolating signal vias from noise sources. The 1D-EBG structure generates about $70 \mathrm{~dB}$ isolation in the path between the noise source and the signal via structure, so as to minimize the coupling of the power/ground cavity noise into the signal lines. In the presence of EBG structures, the voltage noise and timing jitter are significantly reduced for periodic noise sources. However, for random noise sources, the voltage noise and timing jitter can increase in the presence of EBG structures. This paper quantifies this effect.

\section{Introduction}

In recent high speed and high density digital systems, numerous functional blocks are being integrated in a single package using system-in-package ( $\mathrm{SiP}$ ) technologies for achieving system integration. As more chips are integrated in a single package, various noise sources can propagate through the power/ground supply plane. Electromagnetic band gap (EBG) structures can be used as an effective solution for reducing the noise propagation [1],[2]. Since EBG structures can provide isolation in a specific frequency range according to its structure and periodicity, they are effective in reducing the coupled noise through the power/ground planes. However, in a digital system the noise sources can have various spectral contents. This could be a periodic pattern such as the waveforms generated by a clock signal or random noise patterns generated by non-periodic circuit patterns. Unlike the general belief that EBG structures will always reduce the noise coupling, in this paper we quantify the effects of EBG structures where the noise can actually increase depending on the spectral content of the signal. Hence, the EBG structures may not always be beneficial in digital systems.

In this paper, the effect of EBG structures under two different noise source environments are investigated and analyzed. One is a periodic noise source environment and the other is a random noise source environment. In both cases, a signal transition via structure is used as a victim of the noise sources. When a signal experiences multiple reference planes by changing its layers through a signal via structure, the signal quality is degraded by the power/ground noise which is propagated from a noise source and coupled to the via structure [3]. Among various EBG structures, one-dimensional EBG structure (1D-EBG) has been applied to implement the isolation characteristic between the noise source and the victim that is a signal via structure. Figure 1(a) shows the 1DEBG structure which has periodic patterns in $x$-direction. It is implemented using a single layer with the combination of patches and branches which show small and big impedance characteristic respectively [4]. Since the 1D-EBG structure has already been well analyzed to determine its band gap frequency, isolation level, and maximum number of patches [5], this paper focuses not on the characteristic of the EBG structure itself, but on the effect of adopting the EBG structure in various noise source environments. After showing the implementation of the 1D-EBG structure in section II, the different effect of the EBG structure in the presence of 
periodic and random noise is investigated in section III. And the reason is analyzed in section IV.

\section{Implementation of 1D-EBG structure and measurement setup}

Two different types of test vehicles have been implemented to investigate the effect of the 1D-EBG structure on signal transition vias as shown in Figure 1 (a). Both of them have a 4-layer stack-up, and the signal starts from the top-layer and arrives to the bottom layer through one via structure in the middle of the signal path. The only one difference between the test vehicles is on the second layer of the stack-up. One has the form of a 3 pieces $1 D-E B G$ pattern in the second layer as a power plane, and the other test vehicle has the form of a solid plane instead of the EBG pattern. How big different results we can get with the simple different patterns on the second layer is to be verified through the measurement setup shown in Figure 1 (b). The noise source is located at the far edge from the via as shown in Figure 1 (b).

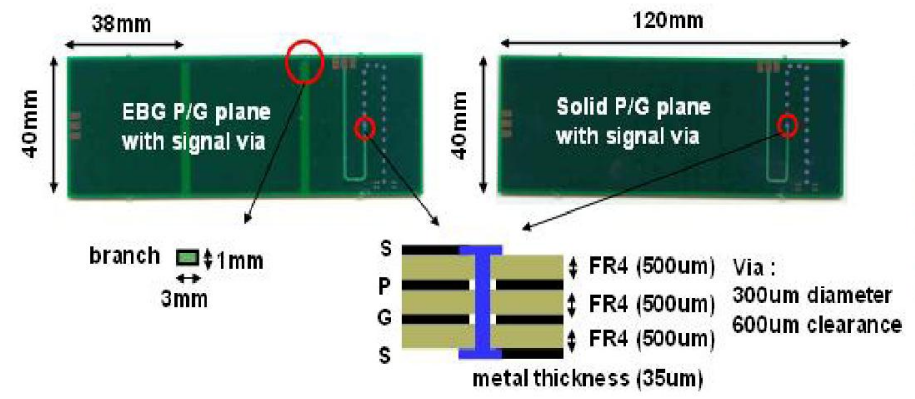

(a)



(b)

Fig. 1. Test DUT's and measurement setup (a) 1D-EBG structure implemented as a power plane under the signal line with a single via at the middle of the signal path (b) Measurement setup to investigate clock jitter and data eye

The 1D-EBG in Figure 1 has been designed to have the band gap frequency from $600 \mathrm{MHz}$ to $1.8 \mathrm{GHz}$. Figure 2 shows both the simulation result and the measurement result of the 1D-EBG structure. Both of them are well matched and show the band gap frequency between $600 \mathrm{MHz}$ and $1.8 \mathrm{GHz}$ with a $-70 \mathrm{~dB}$ isolation level.

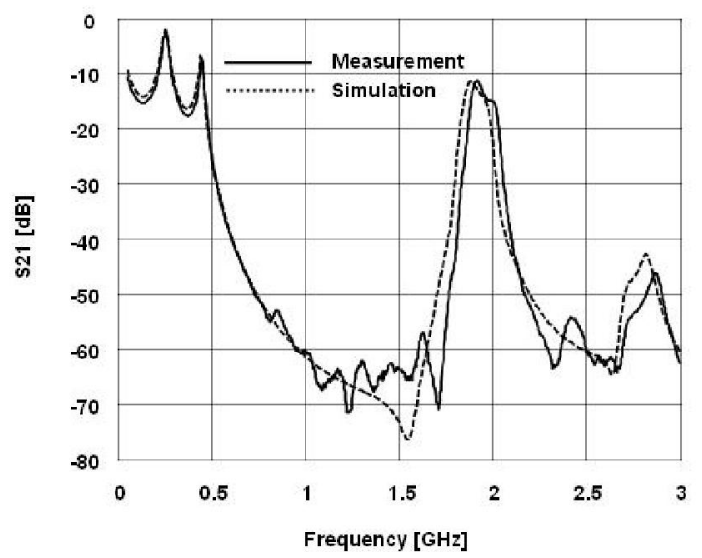

Fig. 2. Simulation and measurement results of 1D-EBG structure

\section{Effect of EBG structure in periodic and random noise source environments}

Figure 3 shows the measurement results of implementing the 1D-EBG structure between the noise source and the via. The noise source used in this experiment is periodic and has a $600 \mathrm{MHz}$ fundamental frequency so that the EBG can mitigate up to the $3^{\text {rd }}$ harmonic frequency of the noise signal. As shown in Figure 
3 (a), a $500 \mathrm{MHz}$ clock signal running on the EBG board generates $180 \mathrm{mV}$ voltage noise and $51.1 \mathrm{ps}$ timing jitter by a single via transition at the middle of the signal path, while the same clock signal running on a general solid board generates $420 \mathrm{mV}$ voltage noise and $74.2 \mathrm{ps}$ timing jitter. Similar results can be acquired by investigating data eye as shown in Figure 3(b). A 500Mbps data signal on the EBG board guarantees $1.58 \mathrm{~V}$ eye opening with 25.3 ps timing jitter, while a general solid board deteriorates the eye opening and the timing jitter into $1.44 \mathrm{~V}$ and $39.4 \mathrm{ps}$ respectively. These results indicate that the EBG structure is effectively able to separate the signal via from a noise source, so as to inhibit the power/ground cavity noise from propagating into the via structure.

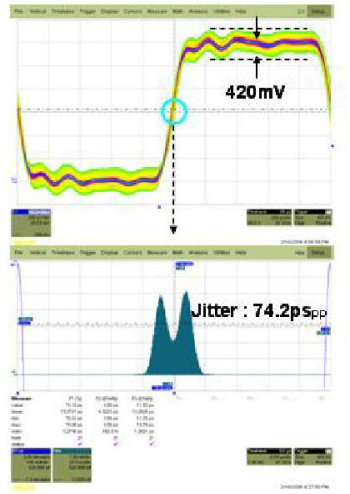

(a) Solid board

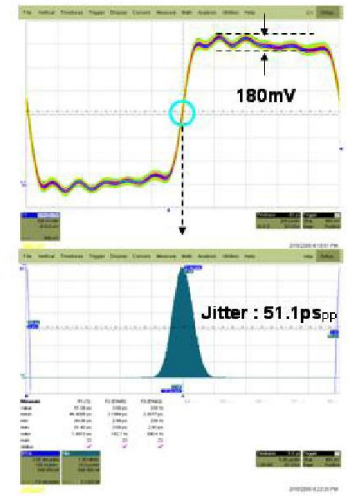

(b EBG board

(a)

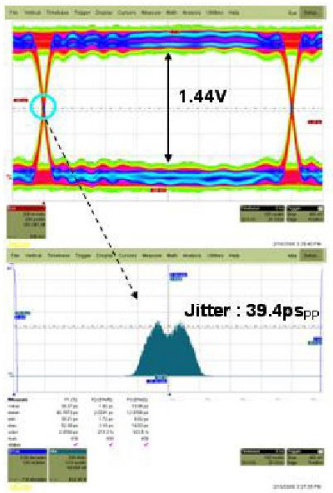

(a) Solid board



(b EBG board

(b)

Fig. 3. Effect of EBG structure in periodic noise; (a) Measurement of $500 \mathrm{MHz} 1.8 \mathrm{~V}$ clock signal under $600 \mathrm{MHz} 400 \mathrm{mV}$ periodic noise; Reduced jitter $=23.1 \mathrm{ps}$ (b) Measurement of $500 \mathrm{Mbps} 1.8 \mathrm{~V}$ data signal under $600 \mathrm{MHz} 400 \mathrm{mV}$ periodic noise; Reduced jitter $=14.1 \mathrm{ps}$

The results in Figure 3 have been acquired from the case of a periodic noise, which means the noise signal is assumed to be generated by periodic operation such as clock signaling. However, the noise source could have a random pattern generated by digital switching noise. In that case, the effect of the EBG is different. As shown in Figure 4 (a), a 500MHz clock signal running on the EBG power plane has worse timing margin than that of the same clock signal on a general solid power plane. Similar results are investigated in eye diagram in Figure 4 (b). The eye opening of the 500Mbps data signal on the EBG power plane shows smaller eye opening and more timing jitter than the case of a solid power plane. These results indicate that the EBG should be carefully implemented to take advantage of it.

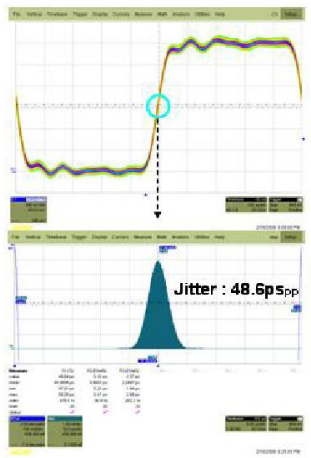

(a) Solid board

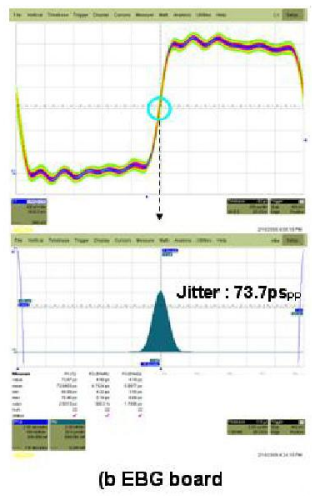

(a)

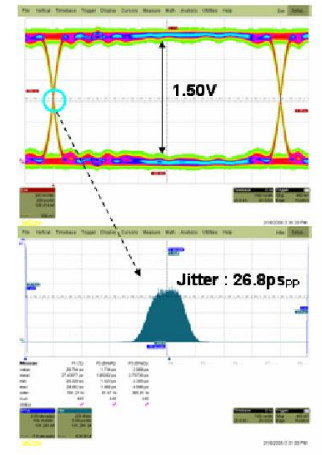

(a) Solid board

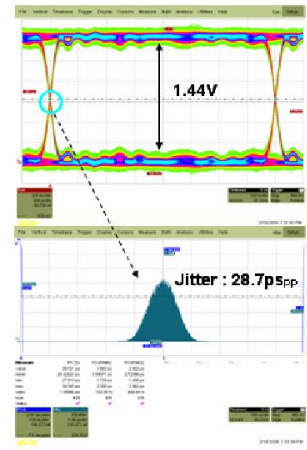

(b) EBG board

(b)

Fig. 4. Effect of EBG structure in random noise; (a) Measurement of $500 \mathrm{MHz} 1.8 \mathrm{~V}$ clock signal under $600 \mathrm{MHz} 400 \mathrm{mV}$ random noise; Increased jitter $=25.1 \mathrm{ps}$ (b) Measurement of $500 \mathrm{Mbps} 1.8 \mathrm{~V}$ data signal under $600 \mathrm{MHz} 400 \mathrm{mV}$ random noise; Increased jitter $=1.9 \mathrm{ps}$ 


\section{Analysis of two different effects of EBG structure in periodic and random noise}

The reason why the same EBG shows different results for periodic noise and a random noise is analyzed in Figure 5. The frequency spectrum of the periodic noise used in the experiment of Figure 3 is well confined to the band gap of the 1D-EBG implemented as a power plane. However, the frequency spectrum of the random noise applied to the experiment in Figure 4 has a widely spread form starting from a dc range. Even if the EBG structure can provide deep isolation in a specific frequency range, the random noise spectrum cannot be confined to the band gap due to the wide spreading characteristic. Especially in a low frequency range, a general solid power plane surpasses the EBG power plane from the view point of a noise transfer characteristic as shown in Figure 5 (c). Since the main portion of the random noise spectrum is concentrated in a low frequency range below $500 \mathrm{MHz}$, the EBG could not mitigate the power/ground cavity noise efficiently with the band gap implemented above $600 \mathrm{MHz}$. Therefore, implementing the EBG structure as a power supply plane can be a good solution not to isolate a random noise but to isolate a periodic noise, unless any other technique compensating for the disadvantage at a low frequency range is adopted along with the EBG technique.

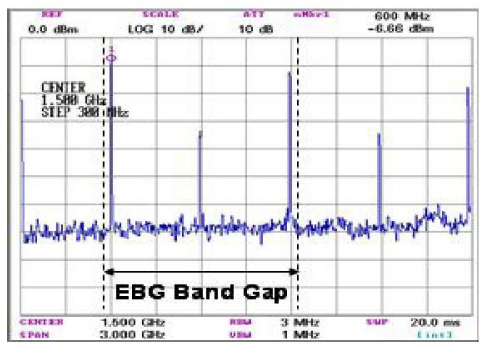

(a)



(b)

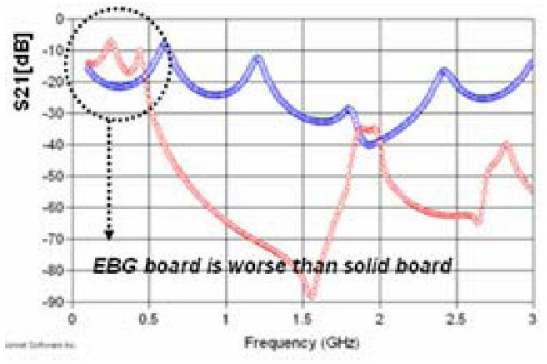

(c)

Fig. 5. Noise Spectrum and EBG characteristic; (a) Frequency spectrum of periodic noise source (b) Frequency spectrum of random noise source (c) Transfer characteristic of EBG and solid board

\section{Conclusion}

In this paper, the effect of EBG structures in periodic and random noise source environments has been investigated. While the EBG structure used in a periodic noise environment guarantees much lower noise coupling and timing jitter, the EBG structure in a random noise environment increased the noise coupling and timing jitter. Since the frequency components of random noise is unknown, it is impossible to make the EBG work for all random patterns. However, for specific busses such as the $1.2 \mathrm{GHz}$ bus, PCI-X etc. where the noise components are known and fall within the band gap, EBG structures will work very well. For periodic noise sources, EBG structures will always work very well.

\section{References}

[1] T. Kamgaing and $O$. Ramahi, "A novel power plane with integrated simultaneous switching noise mitigation capability using high impedance surface", IEEE Microwave and Guided Wave Letters, vol. 13, no. 1, January 2003

[2] R. Abhari and G. V. Eleftheriades, "Metallo-dielectric electromagnetic bandgap structures for suppression and isolation of the parallel-plate noise in high-speed circuits", IEEE Tran. Microwave Theory and Techniques, vol. 51, no. 6, pp. 1629-1639, June 2003

[3] J. Park, at el., "Noise Coupling to Signal Trace and Via from Power/Ground Simultaneous Switching Noise in High Speed Double Data Rates Memory Module", Electromagnetic Compatibility, 2004 IEEE International Symposium on, Volume 2, 9-13, pp. 592-597, Aug. 2004

[4] J. Choi, V. Govind, and M. Swaminathan, "A novel electromagnetic bandgap (EBG) structure for mixed-signal system applications", IEEE Radio and Wireless Conference (RAWCON) 2004, Atlanta, Georgia, pp. 243-246, September 2004

[5] T. Kim, D. Chung, E.Engin, W. Yoon, Y. Toyota, M. Swaminathan, "A Novel Synthesis Method for Designing Electromagnetic Band Gap (EBG) Structures in Packaged Mixed Signal Systems", IEEE Electronic Components and Technology Conference (ECTC), May 2006 